Proceedings of the 2011 Winter Simulation Conference

S. Jain, R.R. Creasey, J. Himmelspach, K.P. White, and M. Fu, eds.

\title{
MULTI-OBJECTIVE COMPASS FOR DISCRETE OPTIMIZATION VIA SIMULATION
}

\author{
Loo Hay Lee \\ Ek Peng Chew \\ Haobin Li \\ National University of Singapore \\ Department of Industrial and Systems Engineering \\ 1 Engineering Drive 2, SINGAPORE 117576
}

\begin{abstract}
Due to its wide application in many industries, discrete optimization via simulation (DOvS) has recently attracted more research interests. As industry systems become more complex, advanced search algorithms for DOvS are desired with higher expectation towards efficiency. In this research work, we combine the ideas of single-objective Convergent Optimization via Most-Promising-Area Stochastic Search (COMPASS) with the concept of Pareto optimality to propose multi-objective (MO) MO-COMPASS for solving DOvS problems with two or more objectives. Numerical experiments are illustrated to show its ability to achieve high efficiency.
\end{abstract}

\section{INTRODUCTION}

It is obvious that, recently, discrete optimization via simulation (DOvS) has had broad application in certain industries such as manufacturing, logistics and services, and is attracting more research interests as well. A simple reason is due to the fact that the performances of these systems largely depend on integer settings like the staffing or inventory level, number of equipments, products or customers. Besides, we noted that in some circumstances, continuous decision variables should also be considered in a discrete sense, for instance, the manufacturing time is usually calculated in number of shifts.

In DOvS problems, quite often the increasing complexity is the key issue of concern and that is usually caused by complicated system designs. To be more specific, as the number of candidate designs is factorized by each design criterion, it increases exponentially as more criteria are taken into consideration. In order to make in-time decisions for industries, DOvS needs more efficient search algorithms.

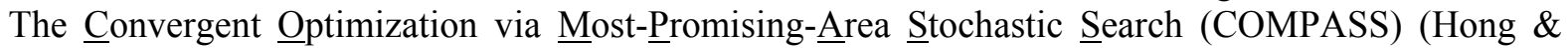
Nelson, 2006) was proposed for this purpose. With this method, solutions are sampled stochastically within the most-promising-area, in which all solutions have shorter Euclidian distance to the current optima than the distance to any current non-optima. The solutions are to be evaluated according to certain simulation allocation rule (SAR) and used to construct the next most-promising-area. It has been proven that the searching converges to the local optima regardless of the searching space being constrained.

However, the COMPASS approach has the limitation that it can only be applied on single-objective problems. It is not sufficient, as nowadays systems often have multiple performance measurements. Although some multi-objective problems can be converted to single-objective by assigning a weightage to each measurement, when there is no consensus about the weightage or in the situation that measurements are not compromising, it will make more sense to provide the Pareto Set, i.e., all non-dominating solutions (Lee et al. 2006, 2008 and 2010) to the decision makers.

A typical multi-objective DOvS application in aerospace industry is D-SIMSPAIR ${ }^{\mathrm{TM}}$, a simulationbased planning and optimization system developed by D-SIMLAB Technologies Pte. Ltd. (Chan et al. 
2006; Lendermann et al. 2010). The main purpose of the system is to provide aircraft spare parts OEM or its designated service provider with the optimal inventory configuration that satisfies targeted service level at minimum cost, by evaluating the performance via simulation. Obviously, the inventory level of each part stored in stock locations are the integer decision variable we can manipulate, and at least two performance criteria, namely the achieved service level (or the probability of achieving target level) and the realized cost, are the objectives we are interested in.

For big airlines or OEM companies, it is common that a flight network contains more than five pool stock locations for a spare part, and each part has an inventory level up to 30 at each location. Clearly, if a full-enumeration scheme is adopted, we need $30^{5}$ trials to visit all possible solutions, which implies that if each visit take 1 second to run the simulation, the optimization process will complete only after 281 days. More severe situation arises when we take more stock locations into consideration, for example, with 6 stock locations, $30^{6}$ visits will takes 23 years. D-SIMSPAIR ${ }^{\mathrm{TM}}$ has been adopting heuristics-based approach to reduce this complexity, hence speeding-up the optimization process. But to bring the capability (scalability in problem size) of D-SIMSPAIR to a new level, a revision to the optimization algorithm is required.

D-SIMSPAIR $^{\mathrm{TM}}$ is one of the example showing a strong call from industries for high efficiency algorithm in solving multi-objective DOvS problems. Since COMPASS works well for single-objective DOvS, this research follows its idea together with the fundamentals of solving multi-objective (MO) problems. We propose a MO-COMPASS algorithm that adapts to multi-objective circumstances and illustrate numerical examples to show its ability in achieving the desired efficiency.

\section{BACKGROUND}

Before explaining the algorithm of MO-COMPASS, it is important to gain some basic knowledge about the working mechanism of single-objective COMPASS and the concept of Pareto optimality.

\subsection{Single-Objective COMPASS}

The introduction of single-objective COMPASS is based on Hong \& Nelson (2006).

For a fully constrained problem, consider the searching space is $\Theta$ and for each $\vec{x} \in \Theta$ the expected single performance measurement is $g(\vec{x})$, which is estimated by aggregated sample average $\bar{G}(\vec{x})$ from simulation results. Without any preliminary knowledge, the most-promising-area $C$ is initially set to $\Theta$.

Let $V$ be the set of all visited solutions. Every iteration, stochastically select $m$ solution from $C$ to be included in $V$ and apply SAR on it to find the solution with the minimum aggregated sample average, and use it to refine the most-promising-area, i.e.,

$$
\begin{gathered}
\hat{\vec{x}}^{*}=\underset{\vec{x} \in V}{\arg \min } \bar{G}(\vec{x}), \\
C=\left\{\vec{x} \mid \vec{x} \in \Theta, \vec{y} \in V, \vec{y} \neq \hat{\vec{x}}^{*},\left\|\vec{x}-\hat{\vec{x}}^{*}\right\| \leq\|\vec{x}-\vec{y}\|\right\} .
\end{gathered}
$$

We should take note that the selection of SAR affects the efficiency of search algorithms, as the search algorithm determines which solutions to visit while the SAR decides how much simulation budget to spend on each visit. According to Hong \& Nelson (2006), a valid SAR for COMPASS should satisfy two conditions: (1) the simulation budget allocated to newly visited solution should not be zero; (2) as total budget approaches infinity, the budget allocation to each visited solution should approach infinity as well.

In $\mathrm{Xu}$, Nelson \& Hong (2010), it is stated that fixed schedules or OCBA (optimal computing budget allocation) ideas can be adopted for COMPASS. Moreover, some SARs are integrated with search algorithm. For example, He et al. (2010) propose an integrated cross-entropy method with OCBA. 
It is proven that repeating the process will lead the estimated $\hat{\bar{x}}^{*}$ converge to the local optima (Hong and Nelson 2007). The searching process can be terminated either when simulation budget is exhausted or all neighboring solutions of $\hat{\bar{x}}^{*}$ are visited. The process can be illustrated by Figure 1 .
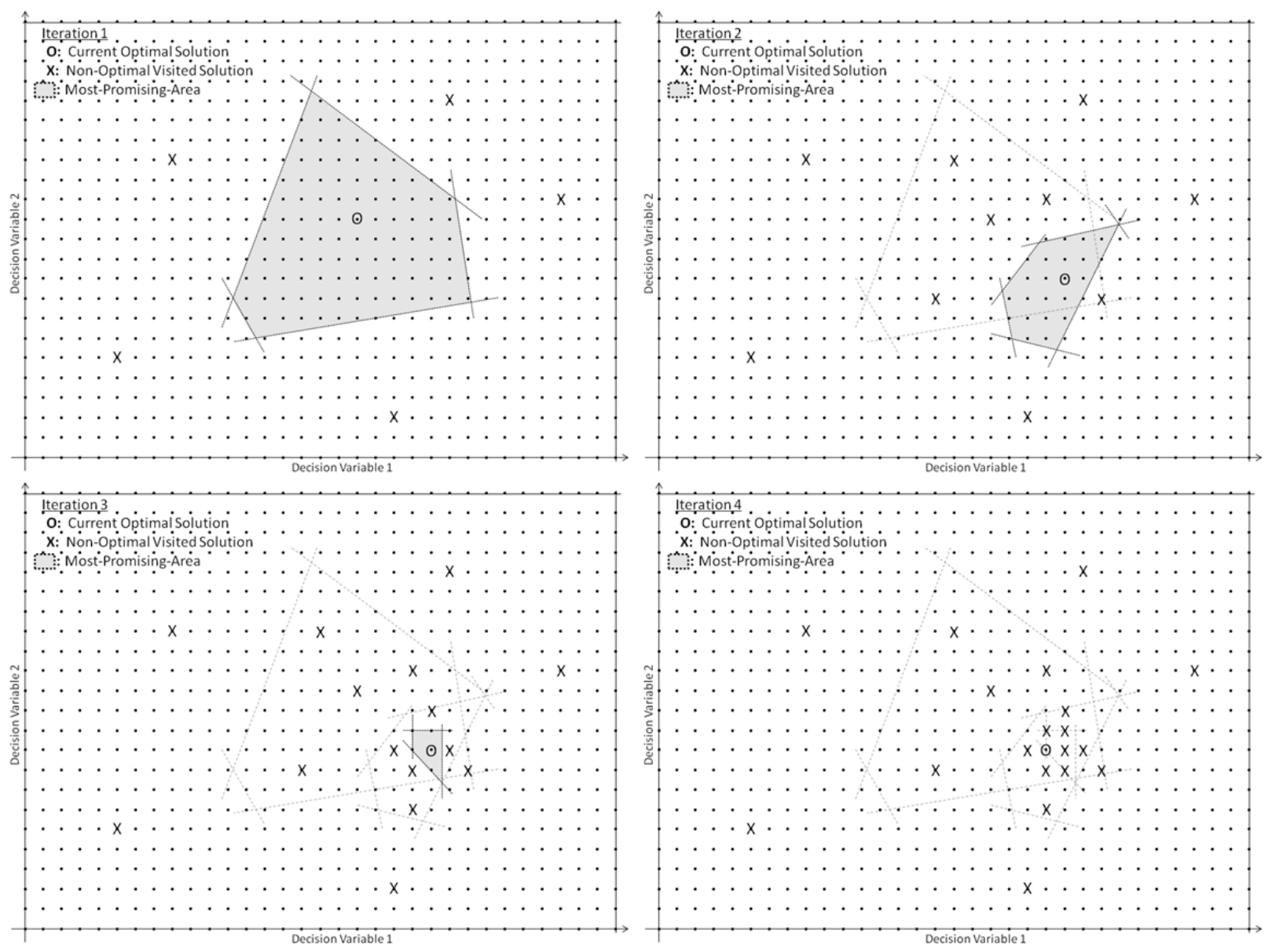

Figure 1: Illustration of single-objective COMPASS

For a partially or unconstrained problem, searching starts from a constrained subset and follows the similar steps as for fully constrained problems, but the boundaries of the subset are revised accordingly for each iteration so as to reserve certain buffer in each direction from $\hat{\bar{x}}^{*}$ when it is available.

\subsection{Pareto Optimality}

To compare two multi-objective solutions, besides looking at the weighted sum of various measurements as a single compromising solution (Butler, Morrice, and Mullarkey 2001; Swisher, Jacobson, and Yücesan 2003), it is more suitable to compare measurement for individual objective (Lee et al. 2006 and 2010). A solution is claimed to dominate another if and only if all its objective measurements are superior to the other's. Thus, in optimization, instead of looking for a single best solution among $\Theta$, we are more interested in finding a set of solutions that are not dominated by the others. We claimed those solutions as best among $\Theta$ and the set is referred as the Pareto set:

$$
\Pi=\Theta \backslash\{\vec{x} \mid \vec{x} \in \Theta, \exists \vec{y} \in \Theta, \vec{g}(\vec{y}) \prec \vec{g}(\vec{x})\}
$$


Similar to single-objective problems, it is difficult to obtain a full Pareto set especially in a huge solution space, since most of the time we are not able to deny the possibility that the current Pareto solution being dominated by some unvisited solutions. Whereas, according to following definition, we can claim a local Pareto optimality without visiting all feasible solutions.

The definition of local Pareto set for a continuous problem has been proposed by Deb (1999), and the definition of local optimality has been adopted by single-objective COMPASS (Hong \& Nelson, 2006). Similarly, we can define local Pareto optimality for a multi-objective discrete problem.

Definition 1 For a discrete problem defined on $\Theta$, a solution set $P \subseteq \Theta$ is claimed as local Pareto set (LPS) if and only if none of its element is dominated by any other solutions in $P$, or any neighboring solutions that has unit Euclidean distance to $P$ (i.e., to the nearest solution in set $P$ ).

Or, in mathematical form we can define LPS as:

$$
P=\{\vec{x} \mid \neg(\exists \vec{y} \in P, \exists \vec{z} \in \Theta,\|\vec{y}-\vec{z}\| \leq 1, \vec{z} \prec \vec{x})\}
$$

An illustration can be shown by the $4^{\text {th }}$ part of Figure 2 (i.e., Iteration $K$ ). As all the circled solutions are incomparable with each other, and all their un-circled neighbors are visited and shown to be nondominating (simply because the circled ones are interim Pareto solutions among all visited), any set of circled solutions can be claimed as a LPS.

However, in multi-objective optimization we usually require the Pareto solutions to be more explorative. Thus we extend the definition of LPS as follows.

Definition 2 For a discrete problem defined on $\Theta$, an LPS $P$ is claimed as closed local Pareto set (CLPS) if and only if each neighboring solution that has unit Euclidean distance to any element of $P$, either belongs to $P$ or be dominated by some solution in $P$.

Again in part 4 of the Figure 2, the set of all circled solutions are claimed as a CLPS as it satisfies the conditions in Definition 2.

Aiming to identity a closed local Pareto set for a multi-objective DOvS with high efficiency, we extend the single-objective COMPASS algorithm to the multi-objective framework.

\section{MO-COMPASS ALGORITHM}

The MO-COMPASS algorithm can be structured following the basic principle of single-objective COMPASS, meaning the most-promising-area is constructed according to Euclidean distances to both "good" and "bad" solutions.

The only difference is that in multi-objective problem, "good" solutions refer to those contained in intermediate Pareto set. Since they are incomparable among each other, the most-promising-areas are constructed for each Pareto solution and are treated indifferently in terms of the chances of sampling new solutions. To be more specific, assuming $\Pi_{k}$ is the Pareto set at iteration $k$ and $V_{k}$ is the set of all visited solutions, the most-promising-area $C_{k}$ is defined as:

$$
C_{k}=\bigcup_{\vec{z} \in \Pi_{k}}\left\{\vec{x} \mid \vec{x} \in \Theta, \vec{y} \in V_{k} \backslash \Pi_{k},\|\vec{x}-\vec{z}\| \leq\|\vec{x}-\vec{y}\|\right\}
$$

Then the algorithm is described as follows: 
Step 0: Let iteration count $k=0, C_{0}=\Theta$ and $V_{0}=\varnothing$.

Step 1: Let $k=k+1$. Sample a set of unvisited solution $X_{k}$ from $C_{k-1}$ with batch size $\left|X_{k}\right|=\min \left(m,\left|C_{k-1} \backslash V_{k-1}\right|\right)$ and let $V_{k}=V_{k-1} \cup X_{k}$. For all solutions in $V_{k}$, apply SAR to collect simulation observations and identify the Pareto set $\Pi_{k}$ accordingly.

Step 2: Based on $\Pi_{k}$ and $V_{k}$, construct $C_{k}$ and go to Step 1 .

Similar to the single-objective COMPASS, the iteration can be repeated until simulation budget is exhausted or the CLPS is identified. It should be noted that, $\Pi_{k}$ is the set of non-dominated soluteons among all visited solutions at any iteration $k$; thus, if all feasible solutions are visited upon termination, the obtained CLPS becomes the global Pareto set (GPS). The algorithm is illustrated by Figure 2.

However, since in MO-OCMPASS we are looking for a set of Pareto solutions instead of a single optima, it is possible that the solutions in the neighborhood of a Pareto solution are also involved in the set. To avoid duplicated sampling we only count newly visited solutions into the batch size $m$, which is different from single-objective COMPASS.
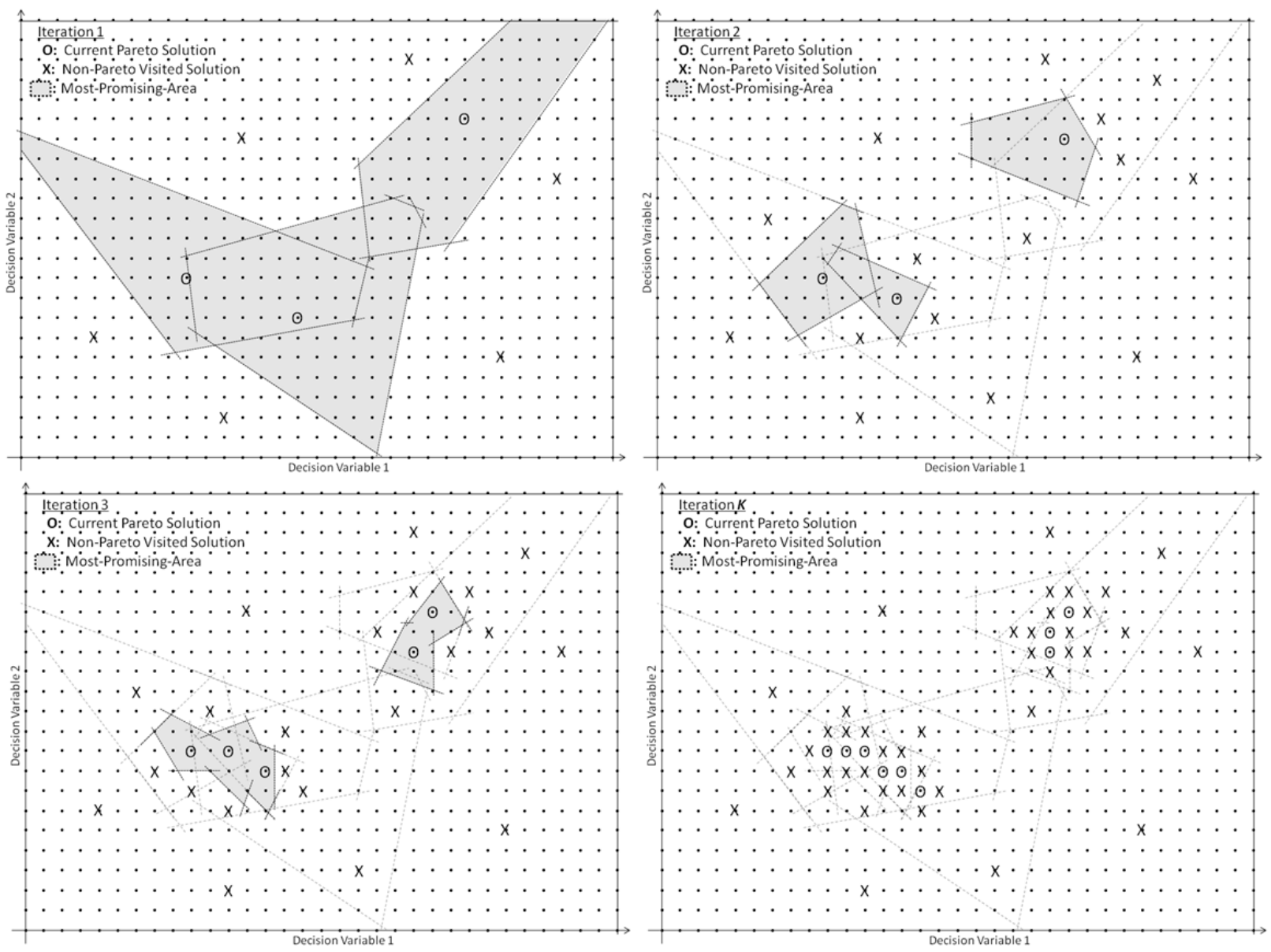

Figure 2: Illustration of MO COMPASS 
The MO-COMPASS is able to apply a method for dealing with partially constrained or unconstrained problems that is similar to single-objective COMPASS, by updating the boundaries of hyper-rectangular searching subset to reserve buffer area for each Pareto solution when it is available.

For random selection from $C_{k}$, Hong \& Nelson (2006) proposed a revised Mix-D (RMD) method, that was only applicable to problems with convex solution set. Since in MO-COMPASS $C_{k}$ can be nonconvex, we divide the selection into two steps: firstly uniformly select a Pareto solution $\vec{z} \in \Pi_{k}$ and then apply $\mathrm{RMD}$ on the subset $P_{k}(\vec{z})=\left\{\vec{x} \mid \vec{x} \in \Theta, \vec{y} \in V_{k} \backslash \Pi_{k},\|\vec{x}-\vec{z}\| \leq\|\vec{x}-\vec{y}\|\right\}$. It is obvious that, $C_{k}=\bigcup_{\vec{z} \in \Pi_{k}} C_{k}(\vec{z})$ and each $C_{k}(\vec{z})$ is a convex set. By doing so, although each $C_{k}(\vec{z})$ has equal probability to be selected, solutions lying in overlapping areas tends to have larger chances. Intuitively, it is consistent with our design principle as multiple approaches to one Pareto solution may imply higher probability of it also being a Pareto solution.

Based on RMD, Xu, Nelson \& Hong (2010) improve the sampling procedure further by reducing redundant linear constraints that form the convex set. The same concept can be adopted for MO-COMPASS.

\section{NUMERICAL EXAMPLE}

\subsection{Multi-Objective Quadratic Problem}

The algorithm can be tested by constructing a multi-objective mathematical problem defined on $Z_{n}^{d}=[0, n]^{d} \rightarrow \mathfrak{R}^{m}$ where each objective is a quadratic function formulated as

$$
f_{i}(\vec{x})=\sum_{j=1}^{d}\left(x_{j}-x_{i j}^{*}\right)^{2} \text { for } 1 \leq i \leq m,
$$

in which $\vec{x}_{i}^{*}$, the true optima for $i$ th objective is selected uniformly in $Z_{n}^{d}$.

Without knowing the formulation but only the returned objective values based on given solution, we apply the MO-COMPASS on $Z_{n}^{d}$. In this experiment, we are testing the algorithm efficiency in term of number of visits before reaching the closed local Pareto set (CLPS, see Definition 2), but not the simulation budget spent on it. So the problem is formulated as deterministic, meaning there is no noise term included.

Besides, according to the problem we constructed, it is easy to observe that even when the number of objectives $m$ remains the same, the size of CLPS increases geometrically as dimension $d$ becomes higher. Thus in the experiment, for easy test and comparison, we control CLPS size as 2 by setting $m=2$ and selecting adjacent $\vec{x}_{0}^{*}$ and $\vec{x}_{1}^{*}$ that satisfies $\left\|\vec{x}_{0}^{*}-\vec{x}_{1}^{*}\right\|=1$, thus CLPS $=\left\{\vec{x}_{0}^{*}, \vec{x}_{1}^{*}\right\}$. Since the CLPS contains all Pareto solutions in the searching space, we note that it is also the GPS.

Varying the dimension $d$ and scalar $n$, we test 30 independent applications of MO-COMPASS by initiating with different random seeds. The average number of visits before reaching the CLPS is shown in Figures 3-4 and Table 1.

Note that the Pareto solution $\vec{x}_{0}^{*}$ and $\vec{x}_{1}^{*}$ are set randomly for each $d$ and $n$, but remains the same across independent MO-COMASS trials. Thus, the overall trend is not biased by the location of CLPS. In addition, the result also indicates that the effect of differing CLPS locations is smaller, as the trend versus $d$ and $n$ can be clearly identified. 


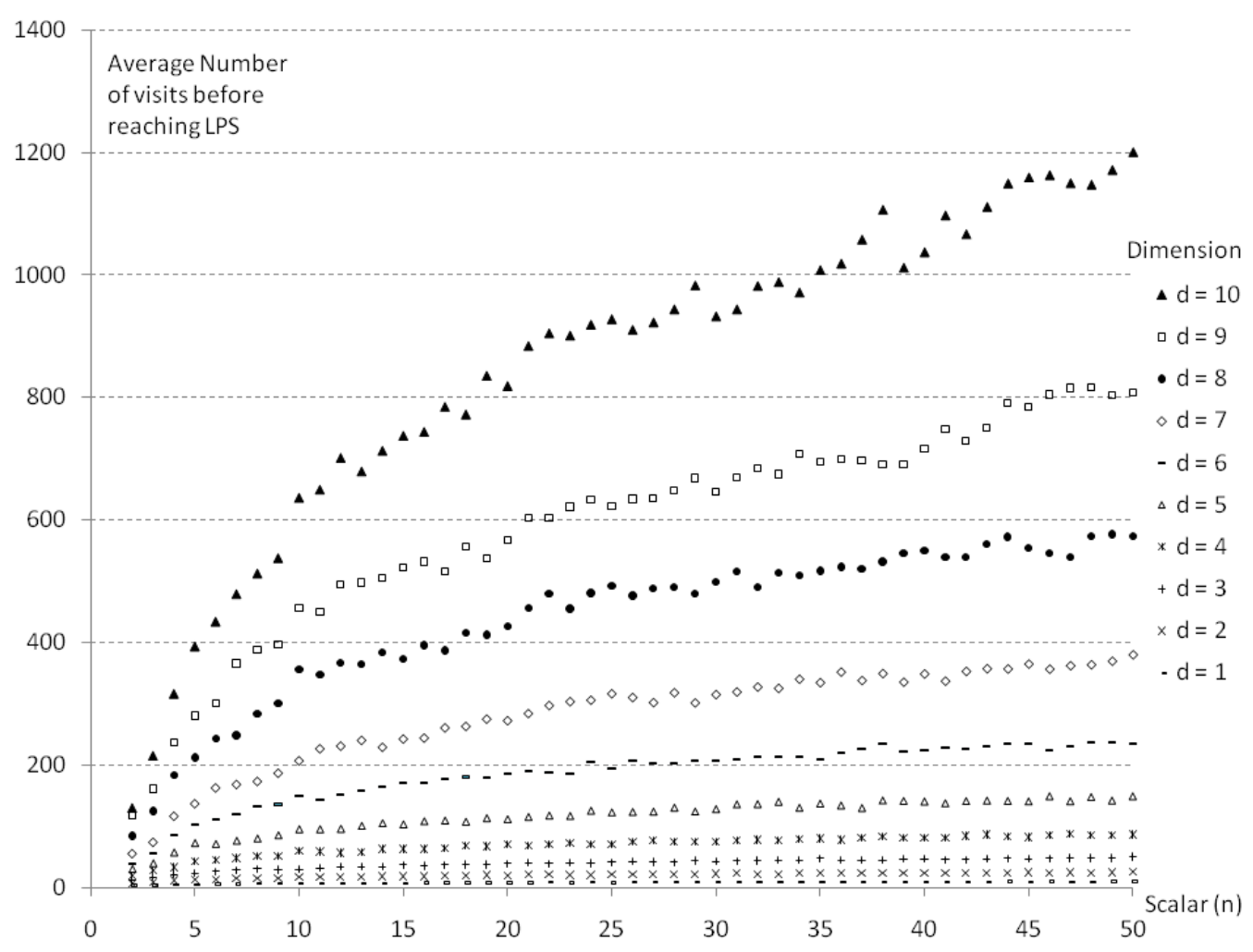

Figure 3: Average number of visits before reaching CLPS, as $n$ varies

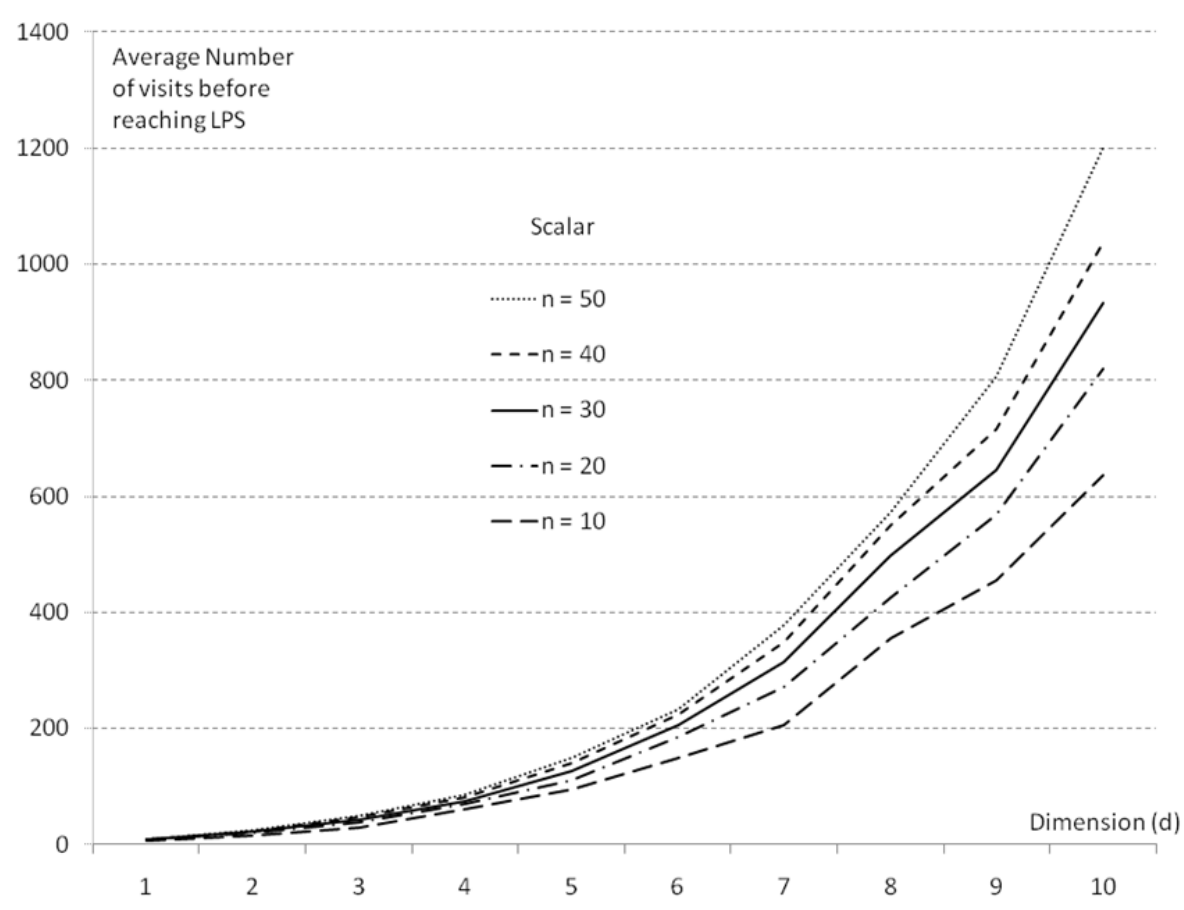

Figure 4: Average number of visits before reaching CLPS, as $d$ varies 
Table 1: Proportion of solutions visited before reaching CLPS, as $d$ varies (\%)

\begin{tabular}{c|cccccccccc} 
& $\mathbf{d}=\mathbf{1}$ & $\mathbf{d}=\mathbf{2}$ & $\mathbf{d}=\mathbf{3}$ & $\mathbf{d}=\mathbf{4}$ & $\mathbf{d}=\mathbf{5}$ & $\mathbf{d}=\mathbf{6}$ & $\mathbf{d}=\mathbf{7}$ & $\mathbf{d}=\mathbf{8}$ & $\mathbf{d}=\mathbf{9}$ & $\mathbf{d}=\mathbf{1 0}$ \\
\hline $\mathbf{n}=\mathbf{1 0}$ & 62.7 & 16.8 & 2.97 & $6.02 \times 10^{-1}$ & $9.47 \times 10^{-2}$ & $1.50 \times 10^{-2}$ & $2.06 \times 10^{-3}$ & $3.56 \times 10^{-4}$ & $4.56 \times 10^{-7}$ & $6.37 \times 10^{-8}$ \\
$\mathbf{n}=\mathbf{2 0}$ & 39.1 & 4.85 & $4.84 \times 10^{-1}$ & $4.37 \times 10^{-2}$ & $3.47 \times 10^{-3}$ & $2.89 \times 10^{-4}$ & $2.12 \times 10^{-5}$ & $1.67 \times 10^{-6}$ & $1.11 \times 10^{-7}$ & $8.00 \times 10^{-9}$ \\
$\mathbf{n}=\mathbf{3 0}$ & 28.9 & 2.51 & $1.57 \times 10^{-1}$ & $9.25 \times 10^{-3}$ & $5.25 \times 10^{-4}$ & $2.84 \times 10^{-5}$ & $1.44 \times 10^{-6}$ & $7.60 \times 10^{-8}$ & $3.28 \times 10^{-9}$ & $1.58 \times 10^{-10}$ \\
$\mathbf{n}=\mathbf{4 0}$ & 22.7 & 1.47 & $7.24 \times 10^{-2}$ & $3.17 \times 10^{-3}$ & $1.37 \times 10^{-4}$ & $5.45 \times 10^{-6}$ & $2.12 \times 10^{-7}$ & $8.39 \times 10^{-9}$ & $2.73 \times 10^{-10}$ & $9.90 \times 10^{-12}$ \\
$\mathbf{n}=\mathbf{5 0}$ & 19.5 & 1.02 & $3.99 \times 10^{-2}$ & $1.38 \times 10^{-3}$ & $4.76 \times 10^{-5}$ & $1.50 \times 10^{-6}$ & $4.85 \times 10^{-8}$ & $1.47 \times 10^{-9}$ & $4.13 \times 10^{-11}$ & $1.23 \times 10^{-12}$
\end{tabular}

From Figure 3 we observe that when dimension remains the same, the number of visits before reaching CLPS increases with the scalar at a rate that is slower than linear. While, if the scalar is kept unchanged, the increasing rate becomes steeper as the dimension is higher (Figure 4). Also, when the size the solution space is taken into consideration, the proportion of visited solutions will converge to zero (Table 1).

Besides, all the results from our numerical settings have shown that, in a deterministic case, MOCOMPASS is able to terminate in a CLPS with finite iterations.

\subsection{Industrial Application}

For testing its ability in solving industrial problems, we apply the MO-COMPASS in D-SIMSPAIR ${ }^{\mathrm{TM}}$ mentioned in Section 1. As there are two phases for its optimization procedure, namely Enumeration and Navigation (Lendermann et al. 2010), our testing addresses only the Enumeration phase that aims to find all best configurations for a part in terms of high service level and low cost. The Navigation phase, which is to form the optimal aggregating plan, is not addressed in this test.

The same simulator incorporated in D-SIMSPAIR ${ }^{\mathrm{TM}}$ was used for evaluating the service level and cost. Besides, a simple SAR was adopted such that each design was only assigned one sufficiently long replication that was ensured to estimate the true performance measures with no error.

As mentioned previously, a heuristic suggested by expert experience is adopted by D-SIMSPAIR ${ }^{\mathrm{TM}}$ to narrow down the searching space. For fair comparison, we incorporate the same heuristic with MOCOMPASS, meaning that if a visited solution is considered as non-promising by the heuristic, we exclude it from any intermediate Pareto sets, without running the simulation.

Table 2: Number of visits and simulations before reaching CLPS for each trial

\begin{tabular}{c|cc}
$\begin{array}{c}\text { Random } \\
\text { Seed }\end{array}$ & $\begin{array}{c}\text { Num. of Visits before } \\
\text { reaching LPS }\end{array}$ & $\begin{array}{c}\text { Num. of Simulations } \\
\text { before reaching LPS }\end{array}$ \\
\hline $\mathbf{0}$ & 642 & 428 \\
$\mathbf{1}$ & 1060 & 297 \\
$\mathbf{2}$ & 985 & 284 \\
$\mathbf{3}$ & 955 & 353 \\
$\mathbf{4}$ & 752 & 195 \\
$\mathbf{5}$ & 1032 & 374 \\
\hline Average & 904 & 322
\end{tabular}


For a testing scenario containing 5 stock locations, 188,109 promising solutions are enumerated and simulated by the current algorithm. Applying six trials with different random seeds, we observe that on average 904 solutions are visited by MO-COMPASS and only 322 are simulated before reaching a CLPS (Table 2). In other words, $0.48 \%$ of current simulation budget is consumed for not incorporating the heuristic, and $0.18 \%$ for doing so.

At the same time, the testing result also shows that it has around $2 / 3$ of chance to obtain the same optimal solutions found by the current method (since a CLPS is not necessarily the global optimal). And for the other $1 / 3$ of times, the difference of the solutions, in terms of minimized cost at the same service level, is less than $0.01 \%$.

\section{CONCLUSION}

In this paper, we explored an advanced search algorithm COMPASS for solving discrete DOvS problem together with some fundamental knowledge for solving multi-objective problems, based on which we proposed an extension for COMPASS to multi-objective circumstances, named as MO-COMPASS.

With numerical example from either mathematical formulation or industry application, we have shown that not only MO-COMPASS is able to converge to a CLPS with finite iterations, it is also capable of solving large scale problems with a high efficiency.

However, current research is under deterministic settings only, as we believe that number of visited solutions would be a good measure when we focus on the search algorithm instead of associated SARs. It may also allow us to compare fairly with other generic multi-objective search algorithms such as MOGAs (Deb 1999) in times to come.

Future research will address the stochastic noise by integrating it with proper SARs, such as MOCBA with indifference-zone (Teng et al. 2010; Lee at al. 2010). Moreover, we may enhance it for a global convergent search, similar to the single-objective industrial strength COMPASS proposed by Xu et al. (2010) for achieving higher convergent rate. With the extended research effort, MO-COMPASS will improve both its accuracy and efficiency in solving DOvS, and gain a wider industrial application.

\section{ACKNOWLEDGMENTS}

The authors thank Dr. Peter Lendermann, Mr. Boon Ping Gan and all staff of D-SIMLAB Pte. Ltd. for their supporting and assistance in this research work.

\section{REFERENCES}

Butler, J., D. J. Morrice, and P. W. Mullarkey. 2001. "A Multiple Attribute Utility Theory Approach to Ranking and Selection." Management Science 47(6): 800-816.

Chan, L. P., K. W. Lye, B. P. Gan, P. Lendermann, and N. Julka. 2006. "Development of a Virtual Warehouse for Aerospace Rotables Management." Singapore Institute of Technology (SIMTech) Technical Report 7(3).

Deb, K. 1999. "Multi-objective Genetic Algorithms: Problem Difficulties and Construction of Test Problems." Evolutionary Computation 7(3):205-230.

He, D., L. H. Lee, C.-H. Chen, M. C. Fu, and S. Wasserkrug. 2010. "Simulation Optimization Using the Cross-Entropy Method with Optimal Computing Budget Allocation." ACM Transactions on Modeling and Computer Simulation 20(1), Article 4, 22 pages.

Hong, L. J., and B. L. Nelson. 2006. "Discrete Optimization Via Simulation Using COMPASS." Operations Research 51(1):115-159.

Hong, L. J., and B. L. Nelson. 2007. "A Framework for Locally Convergent Random-Search Algorithms for Discrete Optimization Via Simulation." ACM Transactions on Modeling and Computer Simulation 17(4), Article 19, 22 pages. 
Lee, L. H., E. P. Chew, S. Teng. 2006. "Integration of Statistical Selection with Search Mechanism for Solving Multi-Objective Simulation-Optimization Problems." In Proceedings of the 2006 Winter Simulation Conference, edited by L. R. Perrone, F. P. Wieland, J. Liu, B. G. Lawson, D. M. Nicol, and R. M. Fujimoto, 294-303. Piscataway, New Jersey: Institute of Electrical and Electronics Engineers, Inc.

Lee, L. H., E. P. Chew, S. Teng, and Y. Chen. 2008. "Multi-Objective Simulation-Based Evolutionary Algorithm for an Aircraft Spare Parts Allocation Problem." European Journal of Operational Research 189(2008):476-491.

Lee, L. H., E. P. Chew, S. Teng, and D. Goldsman. 2010. "Finding the Non-Dominated Pareto Set for Multi-Objective Simulation Models." IIE Transactions 42:656-674.

Lendermann, P., B. P. Gan, N. Julka, A. Schirrmann, and H. Fromm. 2010. "Unlocking Value from Component Exchange Contracts in Aviation Using Simulation-Based Optimization." In Proceedings of the 2010 Winter Simulation Conference, edited by B. Johansson, S. Jain, J. Montoya-Torres, J. Hugan, and E. Yücesan, 2034-2045. Piscataway, New Jersey: Institute of Electrical and Electronics Engineers, Inc.

Swisher, J. R., S. H. Jacobson, and E. Yücesan. 2003. "Discrete-Event Simulation Optimization Using Ranking, Selection, and Multiple Comparison Procedures: A Survey." ACM Transactions on Modeling and Computer Simulation 13(2):134-154.

Teng, S., L. H. Lee, and E.P. Chew. 2010. "Integration of Indifference-Zone with Multi-Objective Computing Budget Allocation." European Journal of Operational Research 203:419-429.

Xu, J., B. L. Nelson, and L. J. Hong. 2010. "Industrial Strength COMPASS: A Comprehensive Algorithm and Software for Optimization Via Simulation." ACM Transactions on Modeling and Computer Simulation 20(1), Article 3, 29 pages.

\section{AUTHOR BIOGRAPHIES}

LOO HAY LEE is an Associate Professor and Deputy Head (Graduate Studies and Research) in the Department of Industrial and Systems Engineering, National University of Singapore. He received his B.S. (Electrical Engineering) degree from the National Taiwan University in 1992 and his S. M. and Ph.D. degrees in 1994 and 1997 from Harvard University. He is currently a senior member of IEEE, a committee member of ORSS, and a member of INFORMS. His research interests include production planning and control, logistics and vehicle routing, supply chain modeling, simulation-based optimization, and evolutionary computation. His email address is iseleelh@nus.edu.sg.

EK PENG CHEW is an Associate Professor and Deputy Head (Undergraduate Studies) in the Department of Industrial and Systems Engineering, National University of Singapore. He received his Ph.D. degree from the Georgia Institute of Technology. His research interests include logistics and inventory management, system modeling and simulation, and system optimization. His email address is isecep@nus.edu.sg.

HAOBIN LI is a Ph.D. candidate in the Department of Industrial and Systems Engineering, National University of Singapore. He received his B.Eng. degree with 1st Class Honors in Industrial and Systems Engineering from National University of Singapore in 2009. His research interests include simulation optimization, and capacity planning problems. He is currently working on an industrial project with DSIMLAB Technologies Pte. Ltd. as a research intern. His email address is li_haobin@nus.edu.sg. 